\title{
Relating Magnetic Properties and High Hyperthermia Performance of Iron Oxide Nanoflowers
}

Bender, Philipp; Fock, Jeppe; Frandsen, Cathrine; Hansen, Mikkel Fougt; Balceris, Christoph; Ludwig, Frank; Posth, Oliver; Wetterskog, Erik; Bogart, Lara K.; Southern, Paul

Total number of authors:

19

Published in:

The Journal of Physical Chemistry Part C

Link to article, DOI:

10.1021/acs.jpcc.7b11255

Publication date:

2018

Document Version

Peer reviewed version

Link back to DTU Orbit

Citation (APA):

Bender, P., Fock, J., Frandsen, C., Hansen, M. F., Balceris, C., Ludwig, F., Posth, O., Wetterskog, E., Bogart, L. K., Southern, P., Szczerba, W., Zeng, L., Witte, K., Gruettner, C., Westphal, F., Honecker, D., González-Alonso, D., Fernández Barquín, L., \& Johansson, C. (2018). Relating Magnetic Properties and High Hyperthermia Performance of Iron Oxide Nanoflowers. The Journal of Physical Chemistry Part C, 122(5), 3068-3077. https://doi.org/10.1021/acs.jpcc.7b11255

\section{General rights}

Copyright and moral rights for the publications made accessible in the public portal are retained by the authors and/or other copyright owners and it is a condition of accessing publications that users recognise and abide by the legal requirements associated with these rights.

- Users may download and print one copy of any publication from the public portal for the purpose of private study or research.

- You may not further distribute the material or use it for any profit-making activity or commercial gain

- You may freely distribute the URL identifying the publication in the public portal 


\section{Relating Magnetic Properties and High Hyperthermia Performance of Iron Oxide}

\section{Nanoflowers}

Philipp Bender, ${ }^{* \dagger} \dagger$ Jeppe Fock, ${ }^{\ddagger}$ Cathrine Frandsen, ${ }^{\ddagger}$ Mikkel F. Hansen, ${ }^{\ddagger}$ Christoph Balceris, "Frank Ludwig, Oliver Posth, Erik Wetterskog," Lara K. Bogart, ${ }^{\perp}$ Paul Southern, ${ }^{\perp}$ Wojciech Szczerba, ${ }^{\#, @ ~ L u n j i e ~ Z e n g, ~}{ }^{\triangle}$ Kerstin Witte,,${ }^{\nabla, \dagger}$ Cordula Grüttner, ${ }^{\dagger \dagger}$ Fritz Westphal, ${ }^{\dagger \dagger}$ Dirk Honecker, ${ }^{\ddagger \dagger}$ David González-Alonso, ${ }^{\dagger}$ Luis

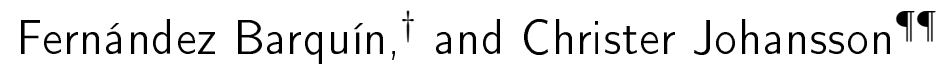

$\dagger$ Universidad de Cantabria, 39005 Santander, Spain.

$\ddagger$ Technical University of Denmark, DK-2800 Kongens Lyngby, Denmark.

๑TU Braunschweig, 38106 Braunschweig, Germany.

$\S$ Physikalisch-Technische Bundesanstalt, 10587 Berlin, Germany.

|| Uppsala University, 75105 Uppsala, Sweden.

$\perp$ University College London, W1S $4 B S$ London, UK.

\#Bundesanstalt für Materialforschung und -prüfung, 12205 Berlin, Germany.

@ AGH University of Science and Technology, 30-059 Krakow, Poland.

$\triangle$ Chalmers University of Technology, 41296 Göteborg, Sweden.

$\nabla$ University of Rostock, 18059 Rostock, Germany.

††Micromod Partikeltechnologie GmbH, 18119 Rostock, Germany.

†Institut Laue-Langevin, 38042 Grenoble, France.

ๆฯRISE Acreo, 40014 Göteborg, Sweden.

E-mail: benderpf@unican.es 


\begin{abstract}
We investigated in depth the interrelations among structure, magnetic properties, relaxation dynamics and magnetic hyperthermia performance of magnetic nanoflowers. The nanoflowers are about $39 \mathrm{~nm}$ in size, and consist of densely packed iron oxide cores. They display a remanent magnetization, which we explain by the exchange coupling between the cores, but we observe indications for internal spin disorder. By polarized small angle neutron scattering we unambiguously confirm that on average the nanoflowers are preferentially magnetized along one direction. The extracted discrete relaxation time distribution of the colloidally dispersed particles indicates the presence of three distinct relaxation contributions. We can explain the two slower processes by Brownian and classical Néel relaxation, respectively. The additionally observed very fast relaxation contributions are attributed by us to the relaxation of the disordered spins within the nanoflowers. Finally, we show that the intrinsic loss power (ILP, magnetic hyperthermia performance) of the nanoflowers measured in colloidal dispersion at high frequency is comparatively large and independent of the viscosity of the surrounding medium. This concurs with our assumption that the observed relaxation in the high frequency range is primarily a result of internal spin relaxation, and probably connected to the disordered spins within the individual nanoflowers.
\end{abstract}

\title{
Introduction
}

Application of iron oxide nanoparticles for magnetic hyperthermia therapy (MHT) has been intensively studied in recent years, as summarized in various review articles. ${ }^{1-4}$ The underlying principle of MHT is to administer nanoparticles within, or close to, tumors, induce heat in the particles by applying an external AC magnetic field, and thereby induce apoptosis of the cells containing magnetic nanoparticles only. ${ }^{5}$ Amongst the ongoing research into the realization of MHT is the optimization of the properties of the nanoparticle ensembles, so as to achieve maximal heating behavior. ${ }^{6-14}$ This in turn is connected to the understanding of 
the dominant heating mechanisms for given particles ensembles, which requires the ability to model experimental results. The simplest model is based on the linear response theory (LRT).

A brief insight into optimization of the particles for increased hyperthermia performance can be gained by considering this theory. Within this theoretical framework, the dissipated power density is given by ${ }^{15}$

$$
P_{d}=\frac{1}{2} \mu_{0} H_{0}^{2} \omega \chi^{\prime \prime}(\omega)
$$

Here, $\mu_{0}$ is the permeability of free space, $H_{0}$ is the amplitude of the alternating excitation field $\tilde{H}(\omega)$ which drives an oscillation of the sample magnetization $\tilde{M}(\omega), \omega=2 \pi f$ is the angular frequency, and $\chi^{\prime \prime}(\omega)$ is the imaginary part of the complex volume susceptibility $\tilde{\chi}(\omega)=\tilde{M}(\omega) / \tilde{H}(\omega)$. For a single relaxation process $\chi^{\prime \prime}(\omega)=\chi_{0} \cdot \omega \tau /\left(1+(\omega \tau)^{2}\right),{ }^{16}$ with the pre-factor $\chi_{0}=M / H$ being the static susceptibility, and $\tau$ being the effective characteristic relaxation time. In case of a single particle it is usually assumed that $\tau=\tau_{\mathrm{N}} \cdot \tau_{\mathrm{B}} /\left(\tau_{\mathrm{N}}+\tau_{\mathrm{B}}\right)$, where:

$$
\tau_{\mathrm{N}}=\tau_{0} \exp \left(\frac{K \pi D_{\mathrm{m}}^{3}}{6 k_{\mathrm{B}} T}\right)
$$

is the classical, longitudinal Néel relaxation time, and:

$$
\tau_{\mathrm{B}}=\frac{\pi \eta D_{\mathrm{h}}^{3}}{2 k_{\mathrm{B}} T}
$$

is the Brownian relaxation time. In Eq. (2), $K$ is the effective anisotropy constant, $D_{\mathrm{m}}$ is the magnetic core diameter, $k_{\mathrm{B}}$ is the Boltzmann constant, and $T$ is the absolute temperature. The pre-factor $\tau_{0}$ is generally taken as $10^{-9}-10^{-10} \mathrm{~s}$, although it is sensitive on several parameters such as the particle size, temperature and dipolar interactions. ${ }^{17,18}$ In Eq. (2), $\eta$ is the viscosity of the surrounding medium and $D_{\mathrm{h}}$ is the hydrodynamic diameter of the particle, including surrounding surfactant and/or depletion layer. Hence, by this theory the key to a large heating power at a given frequency $\omega$ is a large absolute value of the imaginary part of the volume susceptibility. As it stands, clinical applications of nanoparticles are 
restricted to iron-oxide nanoparticles, and so the saturation magnetization is basically fixed, which otherwise could be a valuable approach to increase the heating. ${ }^{19-21}$ Consequently, for a given frequency range an optimization of the MHT performance needs to be achieved by obtaining samples which exhibit significant magnetic relaxation in the frequency range where the MHT experiments are usually performed.

In recent years, so-called nanoflowers, which are densely packed aggregates of iron oxide crystallites/cores, have emerged as promising candidates for MHT. ${ }^{22-25}$ It has been speculated that their phenomenal heating behavior in MHT is primarily a result of an exchange coupling between the cores, which leads to a superferromagnetic magnetization state. ${ }^{24,25}$ With the aim to further advance the understanding of the interrelations between structure, magnetic properties, relaxation dynamics and MHT performance, we performed a detailed study of dextran coated nanoflowers by combining several key techniques.

The results and discussion section consists of four different subsections. It starts with the structural characterization of the nanoflowers, which was mainly conducted by transmission electron microscopy (TEM), dynamic light scattering (DLS) and small-angle X-ray scattering (SAXS). The second part focuses on the magnetic properties of the nanoflowers, which were determined via temperature dependent AC susceptibility $(\mathrm{ACS}(T))$, Mössbauer, isothermal magnetization and field-dependent optomagnetic measurements, as well as polarized small-angle neutron scattering (SANS) experiments. In the third subsection we discuss the relaxation dynamics of the nanoflowers, which was investigated by frequency dependent AC susceptibility $(\operatorname{ACS}(\omega))$, magnetorelaxation (MRX) and frequency-dependent optomagnetic measurements on the colloidally dispersed nanoflowers. And finally in the fourth part we present the MHT results of the colloidal dispersion and discuss them. 


\section{Methods}

The nanoflower particles were supplied by micromod Partikeltechnologie GmbH, Germany. The synomag@-D nanoflowers are dextran coated iron oxide (mixture of $\gamma-\mathrm{Fe}_{2} \mathrm{O}_{3}$ and $\mathrm{Fe}_{3} \mathrm{O}_{4}$ ) nanoparticles, which were synthesized by a polyol method adapted from Lartigue et al. ${ }^{24}$. The concentration of the as-prepared sample was $5 \mathrm{~kg}_{\mathrm{Fe}} / \mathrm{m}^{3}$. Details regarding the synthesis of synomag@particles (research abbreviations: MM08 or NF2) can be found in a previous literature report. ${ }^{26}$

TEM was performed using a JEOL JEM 2100 (FEG). The sample was prepared by drop-casting the diluted dispersion of the particles on a carbon coated copper grid.

DLS measurements of the diluted aqueous dispersion were performed with the Zetasizer Nano ZS90 (Malvern Instr. Ltd., Great Britain). The mean hydrodynamic size (z-average) of the particles was derived from the data of the autocorrelation function by a cumulant analysis.

SAXS of the colloidal dispersion was measured with a Kratky system with slit focus, SAXSess by Anton Paar, Graz, Austria. To obtain the scattering intensity $I(q)$ in absolute units the scattering curves of the sample holder (capillary) and water were subtracted during the data reduction procedure using the implemented SAXSquant software. To additionally correct for the slit focus smearing the curve was deconvoluted with the beam profile.

ICP-OES was conducted with an apparatus from Perkin Elmer, model OPTIME 2100DV, to determine the iron concentration $c_{\mathrm{Fe}}\left[\mathrm{kg}_{\mathrm{Fe}} / \mathrm{m}^{3}\right]$ within the colloidal dispersion.

The $\operatorname{ACS}(T)$ measurements in the range $T=5-390 \mathrm{~K}$ were conducted with a Quantum Design MPMS XL SQUID applying excitation frequencies of $\omega=1.07-5969 \mathrm{~s}^{-1}$. For these measurements the particles were immobilized by putting a small droplet of the colloidal dispersion on cotton wool.

${ }^{57}$ Fe Mössbauer spectroscopy was performed using a conventional constant acceleration spectrometer with a source of ${ }^{57} \mathrm{Co}$ in rhodium. Calibration was carried out at room temperature using a $12.5 \mu \mathrm{m} \alpha$-Fe foil. A closed helium refrigerator from APD Cryogenics 
was used to cool the sample. Approximately $1 \mathrm{ml}$ of the sample $\left(5 \mathrm{~kg}_{\mathrm{Fe}} / \mathrm{m}^{3}\right)$ was added to a measurement capsule and frozen in $\mathrm{N}_{2}(l)$. The spectra were folded and calibrated and the spectra fitted in MATLAB (MathWorks Inc., USA) using a previously described in-house protocol. ${ }^{27}$

Isothermal magnetization curves of the colloidal dispersion were recorded at $T=$ $300 \mathrm{~K}$ using a Magnetic Property Measurement System (MPMS)-XL (Quantum Design, USA). The sample holder $(V=20 \mu \mathrm{l})$ signal was measured and subtracted from all measurements, and additionally the contribution of the water was subtracted from the curve of the colloidal dispersion. The measured magnetic moment was normalized to the iron content $c_{\mathrm{Fe}}$ determined by ICP-OES, to obtain $M(H)$ in units $\left[\mathrm{Am}^{2} / \mathrm{kg}_{\mathrm{Fe}}\right]$.

Polarized SANS was conducted at the instrument D33 at the Institut Laue-Langevin (ILL), Grenoble. ${ }^{28,29}$ Employing longitudinal neutron-spin analysis (POLARIS) ${ }^{30}$ all four neutron spin-resolved intensities $I^{++}(\vec{q}), I^{--}(\vec{q}), I^{+-}(\vec{q})$ and $I^{-+}(\vec{q})$ of the colloidal dispersion were measured in presence of an externally applied, homogeneous magnetic field $\vec{H}$. The field was applied perpendicular to the neutron beam $(\vec{H} \perp \vec{k})$ with field amplitudes of $\mu_{0} H_{\min }=2 \mathrm{mT}$ (minimal field) and $\mu_{0} H_{\max }=1 \mathrm{~T}$ (maximal field). The mean wavelength of the neutrons was $\lambda=0.6 \mathrm{~nm}$, with a wavelength spread of $\Delta \lambda / \lambda=10 \%$ and detector distance $6 \mathrm{~m}$.

MRX experiments were conducted with a custom-built fluxgate setup at $T=300 \mathrm{~K}$, with a measurement time range of $2.8 \cdot 10^{-4}-1.5 \mathrm{~s} .{ }^{31}$ The initially applied field to magnetize the sample was applied for several seconds and had a strength of $2 \mathrm{mT}$.

Frequency-dependent $\operatorname{ACS}(\omega)$ measurements were performed with two custombuilt susceptometers ${ }^{32}$ at $T=300 \mathrm{~K}$ and normalized to obtain the volume susceptibility $\tilde{\chi}(\omega)$. The field amplitudes of the two setups were $567 \mu \mathrm{T}$ and $90 \mu \mathrm{T}$, respectively, and the frequency range $\omega=62.8-6.28 \cdot 10^{6} \mathrm{~s}^{-1}$

Optomagnetic measurements were recorded at room temperature with a custom built cuvette setup described in Ref. 33. The complex second harmonic signal $\tilde{V}_{2}(\omega)=$ 
$V_{2}^{\prime}(\omega)+i V_{2}^{\prime \prime}(\omega)$ in response to a sinusoidal applied magnetic field was calculated using FFT and normalized to the sum of even harmonics, $V_{\text {ref }}=\sum_{i=0} V_{2 i}$, to account for variation in light intensity. For the measurements glycerol was added to the original colloidal dispersion to lower the relaxation frequencies. The weight percentage of glycerol in the mixture was $c_{m}=53.7 \%$ and hence the viscosity was a factor of 7.1 times that of the original aqueous colloid $(\eta=7.1 \mathrm{mPas})$.

Magnetic hyperthermia experiments were performed with a magnetic alternating current hyperthermia 'MACH' system (Resonant Circuits Ltd, UK) using previously described sample preparation and ILP analysis methods. ${ }^{34}$ The viscosity of the colloidal dispersion of nanoflowers was changed by adding glycerol. The applied alternating field had an amplitude of $\mu_{0} H=8.8 \mathrm{mT}$ and a rotational frequency of $\omega=5.9 \cdot 10^{6} \mathrm{~s}^{-1}$.

Numerical inversion approach: Several different approaches exist to extract from experimental data the underlying 1D distribution function, without the need for a priori assumptions regarding their functional form. ${ }^{35-39}$ In Refs. 40,41 for example we used a simple regularized inversion to estimate the moment distribution from isothermal magnetization data. This method is based on the approach introduced in Refs. 42,43 to perform an indirect Fourier transform, which can be used to determine the so-called pair distance distribution functions of nanoparticulate scatterers from small-angle scattering data. In the current work we use the same approach as in Refs. 40,41 to analyze the small angle scattering data of the nanoflowers, their isothermal magnetization measurement as well as the frequencydependent optomagnetic and $\operatorname{ACS}(\omega)$ measurements. But to extract the moment-weighted relaxation time density distribution $p_{\mu}(\tau)$ from $\operatorname{ACS}(\omega)$ data we appended additionally the MRX measurement, which results in more stable solutions of the relaxation time distribution in the low frequency range. The numerical details for the combined inversion of the $\operatorname{ACS}(\omega)$ and MRX measurements can be found in the SI. 
Briefly, the least square solution of the the functional

$$
\left\|\left[\begin{array}{c}
1 / \sigma \cdot \mathbf{U} \\
1 / \sigma \cdot \mathbf{V} \\
1 / \sigma \cdot \mathbf{W} \\
\sqrt{2 \alpha} \mathbf{L}
\end{array}\right] P(\tau)-\left[\begin{array}{c}
1 / \sigma \cdot \chi^{\prime}(\omega) \\
1 / \sigma \cdot \chi^{\prime \prime}(\omega) \\
1 / \sigma \cdot B(t) / c \\
0_{N, 1}
\end{array}\right]\right\|^{2}
$$

was determined for $P(\tau)=\chi_{0} p_{\mu}(\tau) \Delta \tau \geq 0$, where $p_{\mu}(\tau)$ is the moment-weighted relaxation time density distribution $\left(\sum p_{\mu}(\tau) \mathrm{d} \tau=1\right)$. Here, $\sigma$ are the measurement uncertainties, the matrix $\mathbf{L}$ is a $N \times N$ regularization matrix weighted by the regularization parameter $\alpha$ and the vector $0_{N, 1}$ is a zero vector of length $N$, with $N$ being the number of bins of the reconstructed histogram $P(\tau)$. The matrices $\mathbf{U}, \mathbf{V}$ and $\mathbf{W}$ are the $M \times N$ data transfer matrices, with $M$ being the number of data points $\left(U_{i, j}=1 /\left(1+\left(\omega_{i} \tau_{j}\right)^{2}\right), V_{i, j}=\omega_{i} \tau_{j} /\left(1+\left(\omega_{i} \tau_{j}\right)^{2}\right), W_{i, j}=\right.$ $\left.\exp \left(-t_{i} / \tau_{j}\right)\right)$. The constant $c$ is the normalization constant of the MRX signal, which has to be introduced due to the fact that the raw signal of the fluxgate sensors was analyzed.

The least square solution of Eq. (4) was then determined for 200 different values of the regularization parameter $\alpha$ as well as varying values of the normalization constant $c$ of the MRX signal. Afterwards the posterior probability $P(\alpha, c)$ was calculated. The correct value for $c$ was defined as the value $c_{\max }$ for which the highest evidence $P(\alpha, c)$ was calculated. To determine the average distribution $P(\tau)$, all 200 solutions $P_{\alpha}(\tau)$, which were calculated at this particular value $c_{\max }$, were added with a weight given by the probability $P(\alpha)$.

It has to be remarked that the total measurement uncertainty $\sigma$ can assumed to be larger than the standard deviation calculated from repeated measurements. Therefore we used for the numerical inversion of the $M(H)$, otpomagnetic, $\operatorname{ACS}(\omega)$ and MRX measurements in each case an uncertainty of $1 \%$ of the maximal data value. For example in case of the $M(H)$ curve the uncertainty of each data point is assumed to be $0.01 \cdot M_{\mathrm{S}}$. The applied error bars are always plotted but are barely larger than the used symbol size. 


\section{Results and Discussion}

\section{Structural characterization of the nanoflowers}
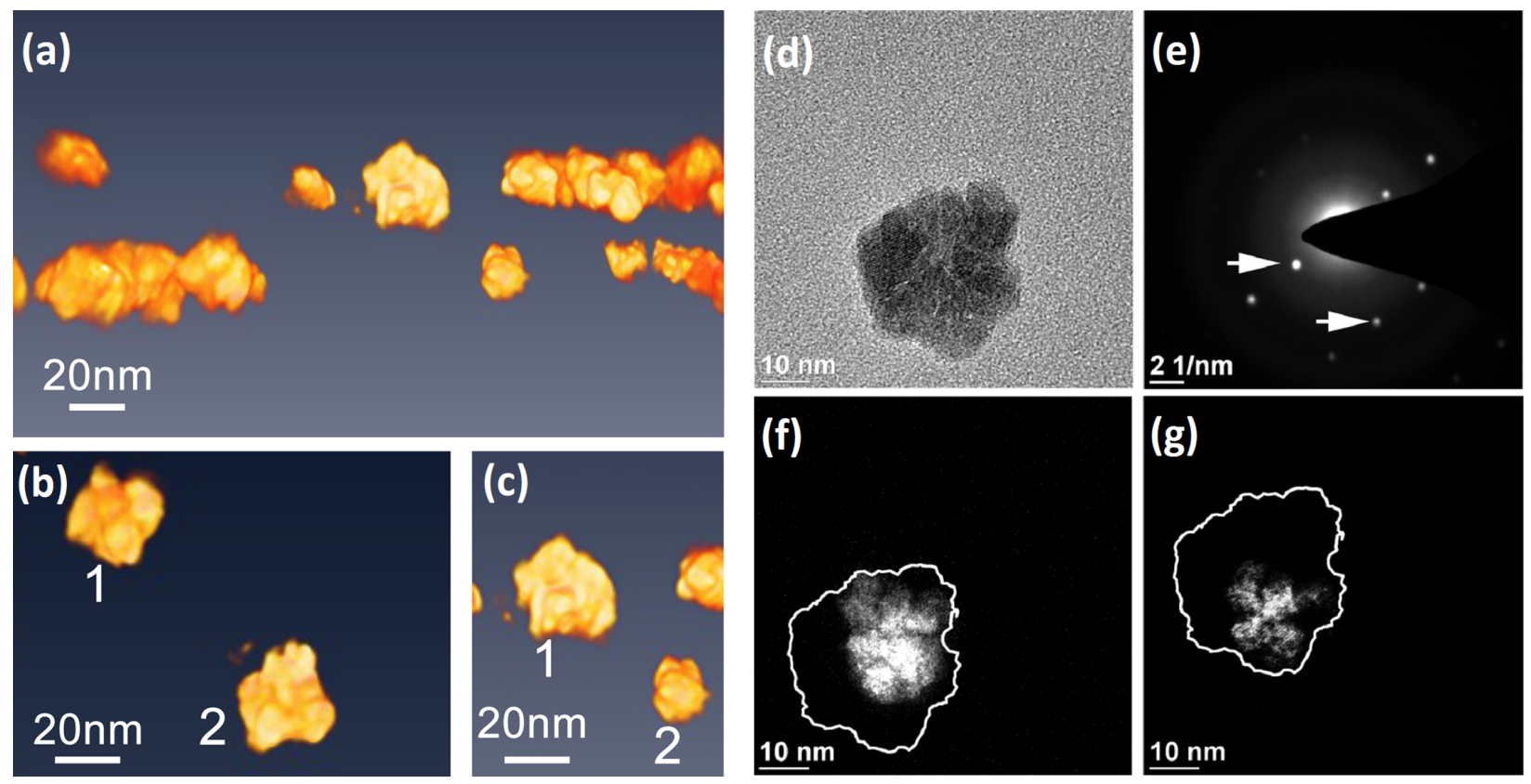

Figure 1: (a) TEM tomography image of the nanoflowers. (b) A closer look at two particles viewed parallel to the electron beam direction in TEM and (c) perpendicular to it. (d) TEM bright field image of a nanoflower. (e) Selected area electron diffraction pattern of the particle shown in $(d)$. Arrows indicate the diffraction spots used to form dark field images shown in (f) and (g).

Figs. 1(a), (b) and (c) show representative TEM tomography images of the nanoparticles. The corresponding video can be found in the supplementary information (SI). The particle sizes were determined from the bright-field TEM images (Fig. 1(d)). By measuring the size of 200 nanoparticles and fitting the histogram with a lognormal function we obtained a numberweighted mean value for the size of $\left\langle D_{N}\right\rangle=39.0 \pm 0.3 \mathrm{~nm}$ and width of $\sigma=0.12 \pm 0.01$. Direct inspection of the particles shows, however, that their shape significantly deviates from homogeneous isometric spheres. We determined the volume of 10 particles directly from the 3D TEM tomography images, which resulted in an average volume of $1.4 \pm 0.5 \cdot 10^{-23} \mathrm{~m}^{3}$. This is about 2.2 times smaller than the volume for spheres with $D=39 \mathrm{~nm}$. The discrepancy can be attributed to the irregular shape of the particles, in particular to their rough surface. Due 
to their characteristic morphology, these particles are commonly referred to as nanoflowers. ${ }^{24}$ Additional investigation indicates a slight anisotropy for most of the particles, as exemplified in Figs.1(b) and (c) for two particles. Their irregular shape is a result of the fact the particles are composed of several small crystallites/cores, which are fused together, as shown in Fig. 1(e), (f) and (g). According to the dark-field TEM images, the volumes with identical crystal orientation are quite diffuse, with sizes ranging from about $5-15 \mathrm{~nm}$. Hence, the particles can be regarded as nanoparticles with a nanocrystalline substructure.

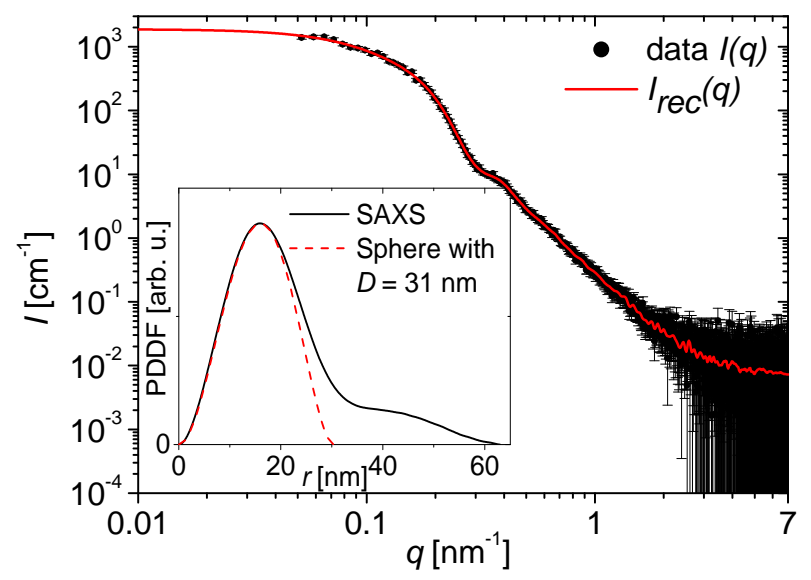

Figure 2: SAXS intensity $I(q)$ measured at $T=300 \mathrm{~K}$ of the colloidal dispersion and reconstructed curve $I_{\text {rec }}(q)$ for the extracted PDDF (fit). Inset: The PDDF determined by an indirect Fourier transform of the scattering intensity $I(q)$ and comparison with the PDDF calculated for a sphere with a diameter of $31 \mathrm{~nm}$.

With DLS a mean hydrodynamic size (z-average) of the particles of $\left\langle D_{\mathrm{h}}\right\rangle=56 \mathrm{~nm}$ was determined, with a polydispersity index of PDI $=0.099$. The hydrodynamic size is significantly larger than the size of the individual nanoflowers determined by TEM (39 nm), and can be explained by the Dextran shell surrounding the nanoflowers which is not visible in TEM.

With SAXS we measured the radially averaged scattering intensity $I(q)$ (Fig. 2), and extracted the underlying radial pair distance distribution function (PDDF) by an indirect Fourier transform, ${ }^{42-44}$ following the approach described in Ref. 40. The PDDF represents the probability of finding a scatterer a distance $r$ away and hence contains information about the average particle geometry. ${ }^{45}$ As seen in Fig. 2 (inset), the derived PDDF exhibits 
a main peak in the low $r$-range with a maximum at $r=16 \mathrm{~nm}$, and finite values in the large $r$-range up to $63 \mathrm{~nm}$. In case of a homogeneous sphere a maximum at $r=16 \mathrm{~nm}$ corresponds to a diamater of $31 \mathrm{~nm} .{ }^{40}$ Accordingly, the main peak is quite well described by the PDDF calculated for a homogeneous sphere with diameter $D=31 \mathrm{~nm}$. The value for the particle diameter is below the average size of the nanoflowers determined by TEM $\left(D_{\mathrm{N}}=39 \mathrm{~nm}\right)$, though, it is safe to assume that this discrepancy can be attributed to the significant deviation of the particles from perfect spheres.

The finite values of the PDDF in SAXS for $r>39 \mathrm{~nm}$ in Fig. 2 then indicate the presence of particle agglomerates. However, the PDDF values for $r>39 \mathrm{~nm}$ are quite low (low probabilities), and we can thus conclude that only a small fraction of the nanoflowers were agglomerated to e.g. dimer-like structures and that the majority of the nanoflowers were individually dispersed.

\section{Magnetic properties of the nanoflowers}

Fig. 3(a) shows the real and imaginary parts, $\chi^{\prime}$ and $\chi^{\prime \prime}$, of $\operatorname{ACS}(T)$ measurements on the immobilized particles, performed at $(T=5-390 \mathrm{~K})$ with varying excitation frequencies $(\omega=$ $\left.1.07-5969 \mathrm{~s}^{-1}\right)$. The imaginary part $\chi^{\prime \prime}$ has a non-zero signal over the whole temperature range, which indicates a broad distribution of blocking temperatures. There are at least

two sets of frequency dependent cusps in both $\chi^{\prime}$ and $\chi^{\prime \prime}$, one indicated by arrows at $300 \mathrm{~K}$, and one above the maximum temperature of the instrument with blocking temperatures $T_{\mathrm{B}}(\omega)>400 \mathrm{~K}$. Considering that the nanoflowers consist of small iron-oxide cores, which are fused together, we speculate that the observed multimodality is a result of a superposition of individual and collective relaxation modes. This suggests the presence of strong interactions between the neighboring nanocrystals within the nanoflowers.

Fig. 3(b) displays the Mössbauer spectrum obtained at $T=18 \mathrm{~K}$ of the frozen dispersion of nanoflowers. As shown in the SI, all spectra are magnetically split (i.e. magnetically blocked) up to at least $200 \mathrm{~K}$. Moreover, the spectra are characteristic of maghemite 

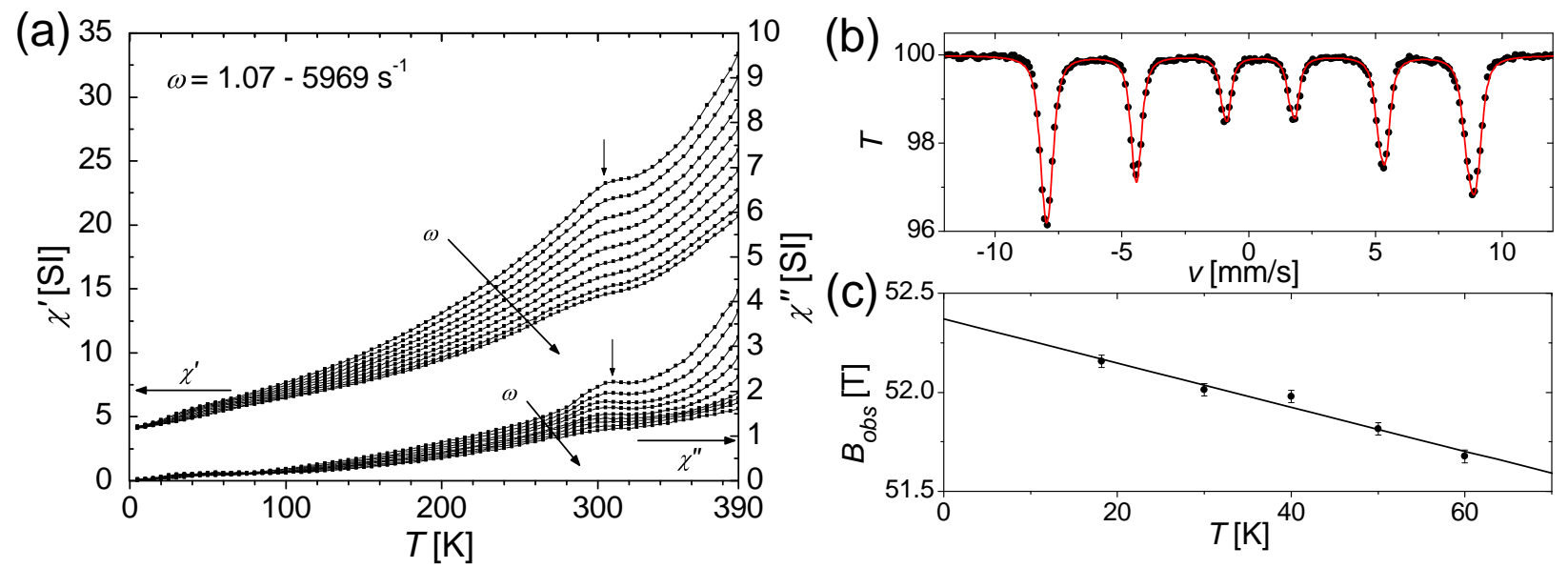

Figure 3: (a) $\operatorname{ACS}(T)$ : Magnitude of real and imaginary parts, $\chi^{\prime}$ and $\chi^{\prime \prime}$, of the susceptibility for varying excitation frequencies $\omega$ measured at different temperatures of the immobilized nanoflowers. (b) Mössbauer: Spectrum obtained at $T=18 \mathrm{~K}$ of the frozen colloidal dispersion and (c) the mean hyperfine field vs. temperature (bottom).

$\left(\gamma-\mathrm{Fe}_{2} \mathrm{O}_{3}\right)$ and the mean isomer shift (calculated from the isomer shifts listed in the SI, according to the method outlined $\mathrm{in}^{46}$ ) shows that the nanoflowers are essentially free of magnetite $\left(\mathrm{Fe}_{3} \mathrm{O}_{4}\right.$ content $\left.5 \pm 5 \%\right)$. At temperatures well below the superparamagnetic blocking temperature, the intra-well fluctuations of the magnetic moment can be measured using Mössbauer spectroscopy due to a reduction of the observed magnetic hyperfine field according to $B_{\mathrm{obs}}=B(T=0 \mathrm{~K})\left[1-k_{\mathrm{B}} T /(\kappa V)\right]$, where $\kappa$ is a constant describing the curvature of the anisotropy energy of a particle core near an energy minimum. ${ }^{47}$ From the analysis of Mössbauer spectra measured between $T=18 \mathrm{~K}$ and $60 \mathrm{~K}$, we obtained $\kappa V / k_{\mathrm{B}}=4700 \pm 300 \mathrm{~K}$ (Fig. 3(c)). For non-interacting particles with uniaxial anisotropy, $\kappa V=2 K V$. In the literature, the anisotropy constant $K$ for maghemite particles larger than $7 \mathrm{~nm}$ has been found to be in the range $1-13 \mathrm{~kJ} \mathrm{~m}^{-3} \cdot{ }^{48-53}$ Due to shape and surface anisotropies, these values are an order of magnitude larger than the bulk value. Assuming a core size of $15 \mathrm{~nm}$ and the upper limit of $K$ we obtain $2 K V / k_{\mathrm{B}}=3400 \mathrm{~K}$, which is well below the measured value of $\kappa V / k_{\mathrm{B}}$. In a simple model, Mørup showed that the presence of exchange coupling between particles serves to increase the curvature of the anisotropy energy and hence result in a value of $\kappa \cdot{ }^{47}$ Indeed, even when considering a combination of magnetocrystalline anisotropy, as well as 
both shape and size anisotropies, we observe a higher than expected value for $\kappa$. Whilst it is not possible to exclude other contributions such as magnetostatic based dipole interactions, our observed value of $\kappa$ is consistent with exchange coupled cores.
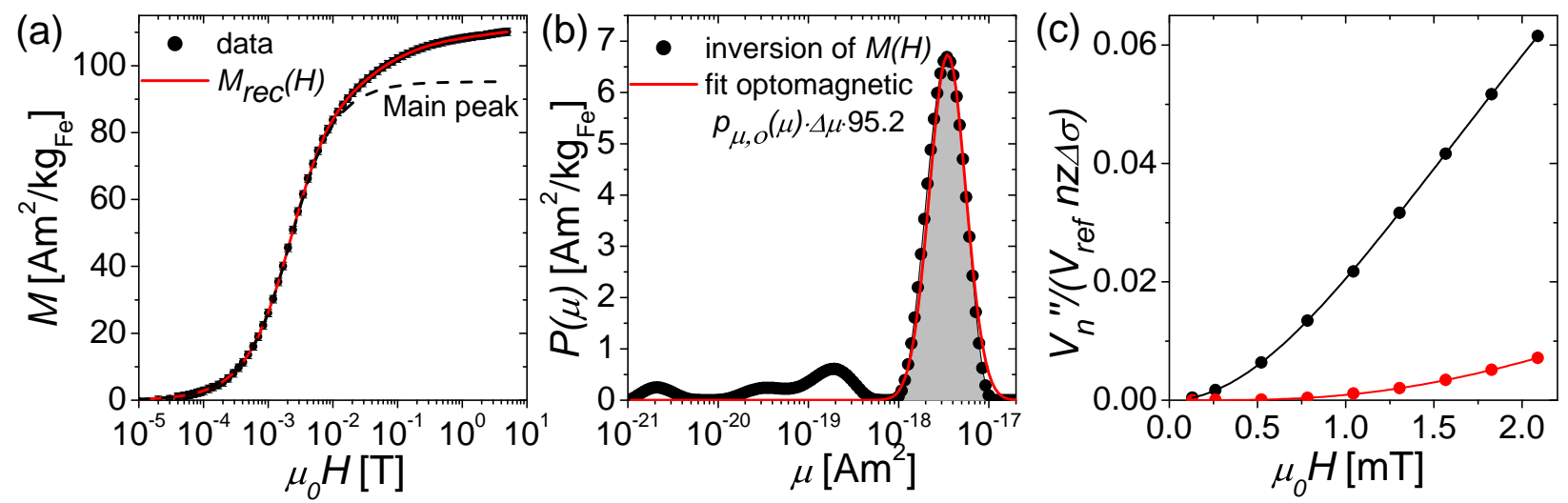

Figure 4: (a) $M(H)$ curve of the colloidal dispersion at $T=300 \mathrm{~K} . \quad M_{\mathrm{rec}}(H)$ is the reconstructed curve for the derived moment distribution $P(\mu)$. Dashed line: Reconstructed magnetization curve using only the main peak of $P(\mu)$. (b) Discrete moment distribution $P(\mu)=M_{\mathrm{S}} p_{\mu}(\mu) \Delta \mu$ (logarithmic spacing $\Delta \mu$, moment-weighted) determined by numerical inversion of $M(H)$ of the colloidal dispersion. Summation of the main peak (grey area) results in a value of $M_{S}^{\text {main }}=\sum_{i=1}^{N} P\left(\mu_{i}\right)=95 \mathrm{Am}^{2} / \mathrm{kg}_{\mathrm{Fe}}$. The red curve is the moment distribution derived from the optomagnetic measurements. (c) Second $V_{2}$ (black) and fourth $V_{4}$ (red) harmonic of the optomagnetic signal measured at $\omega=19 \mathrm{~s}^{-1}, T=300 \mathrm{~K}$ and normalized with the fitted signal strength, $n z \Delta \sigma$, described by the number concentration, $n$, the path length of the light, $z=2 \mathrm{~mm}$, and the difference in extinction cross sections between the particle moment being parallel or perpendicular to the direction of the magnetic field/light, $\Delta \sigma$. The lines are fits to the equilibrium model.

Fig. 4(a) shows the isothermal magnetization curve of the colloidal dispersion measured at $T=300 \mathrm{~K}$ with a logarithmic field scale. The curve is anhysteretic and approaches at $\mu_{0} H=4.9 \mathrm{~T}$ a saturation value of about $M_{\mathrm{S}}=110 \mathrm{Am}^{2} / \mathrm{kg}_{\mathrm{Fe}}$, which is close to the expected value for bulk maghemite $\left(M_{\mathrm{S}}=118 \mathrm{Am}^{2} / \mathrm{kg}_{\mathrm{Fe}}\right) .{ }^{54}$ Assuming the stoichiometry of maghemite this corresponds to a volume magnetization of $M_{\mathrm{S}, \mathrm{V}}=3.74 \cdot 10^{5} \mathrm{~A} / \mathrm{m}$.

To determine the underlying apparent magnetic moment distribution of the nanoflowers we applied a numerical inversion approach using the Langevin-function as model function. ${ }^{40,41}$ Fig. 4(b) shows the extracted moment-weighted distribution $P(\mu)=M_{\mathrm{S}} p_{\mu}(\mu) \Delta \mu$ (where $p_{\mu}(\mu)$ is the moment-weighted probability density), and the corresponding recon- 
structed curve $M_{\text {rec }}(H)$ is displayed in Fig. 4(a). As can be seen, $P(\mu)$ exhibits one distinct, lognormal-like main peak in the range $\mu=10^{-18}-10^{-17} \mathrm{Am}^{2}$. Using this main peak to reconstruct the magnetization curve yields a value of $M_{S}^{\text {main }}=\sum_{i=1}^{N} P\left(\mu_{i}\right)=95 \mathrm{Am}^{2} / \mathrm{kg}_{\mathrm{Fe}}$, and which provides an excellent description of the observed magnetization behavior for fields $\mu_{0} H<10 \mathrm{mT}$. Therefore, we may conclude that this peak corresponds to the remanent moment distribution of the nanoflowers, which in colloidal dispersion can align along the field direction by Brownian rotation.

Using optomagnetic measurements, the remanent moment distribution of the nanoflowers could be alternatively obtained as described by Fock et al. ${ }^{33}$. Briefly, the magnetic fieldinduced modulation of light transmitted through a suspension of the magnetic nanoparticles was measured vs. the amplitude of an oscillating magnetic field at low frequency. Fig. 4(c) shows the obtained 2'nd and 4'th harmonic components of the transmitted light, and the curves show a fit obtained using an equilibrium model assuming a lognormal distribution of moments. ${ }^{33}$ The optomagnetic signal depends on the scaling of both the magnetic moment and the extinction cross section with the particle size. If both the magnetic moment and the extinction cross sections are proportional to the particle volume, the optomagnetic signal is weighted by the particle volume (or equivalently, the magnetic moment). ${ }^{33}$ With this assumption, the obtained moment (or volume)-weighted magnetic moment distribution $p_{\mu, \mathrm{o}}(\mu)$, shown in Fig. 4(b), had a median moment of $\mu=3.5 \cdot 10^{-18} \mathrm{Am}^{2}$ and $\sigma=0.46$. As can be seen (Fig.4(b)), this distribution is in very good agreement with the main peak of the moment distribution derived from the $M(H)$ curve of the colloidal dispersion. Thus the isothermal magnetization and optomagnetic measurements indicate that the nanoflowers have a moment-weighted mean remanent moment of about $\langle\mu\rangle=3.9 \cdot 10^{-18} \mathrm{Am}^{2}$. This corresponds to a mean volume of $\langle V\rangle=\langle\mu\rangle / M_{S, V} \approx 1 \cdot 10^{-23} \mathrm{~m}^{3}$, which is slightly smaller than the average volume determined by $3 \mathrm{D}$ TEM tomography $\left(1.4 \pm 0.5 \cdot 10^{-23} \mathrm{~m}^{3}\right)$. This indicates a slight spin disorder within the individual nanoflowers, which would reduce the remanent moment. Such an assumption is strengthened by the isothermal magnetization 
behavior detected at high fields. As shown in Fig.4(a), the particle moments should be completely aligned along the field direction at $\mu_{0} H=0.1-1 \mathrm{~T}$ (main peak). By contrast field strengths of more than $\mu_{0} H=5 \mathrm{~T}$ are needed to magnetically saturate the sample. As a consequence of the increasing magnetization in high fields, the apparent moment distribution extracted from the magnetization curve exhibits minor peaks in the low-moment range (Fig. 4(b)). We assume that these contributions belong to uncorrelated/disordered spins within the individual nanoflowers (e.g. surface spins), which account for a volume fraction of $\phi=\left(M_{S}-M_{S}^{\text {main }}\right) / M_{S} \approx 0.2$. Nevertheless, we can surmise that the majority of spins was preferentially magnetized along one direction, which could be unambiguously confirmed by a model independent analysis of polarized SANS data.

The analysis of the polarized SANS experiments focused mainly on the structural information, which is contained in the non-spin-flip (nsf) intensities $I^{--}(\vec{q})$ and $I^{++}(\vec{q})$. The analysis of the nsf channels allows to extract the pure nuclear scattering signal from sectors along the field direction. Defining $x$ as the direction of the neutron beam and $z$ as the direction of the applied magnetic field, the nsf cross sections can be written ${ }^{30,55}$

$$
\begin{aligned}
I^{ \pm \pm}(\vec{q}) \propto & |\tilde{N}|^{2}+b_{\mathrm{h}}^{2}\left|\tilde{M}_{z}\right|^{2} \sin ^{4} \Theta \\
& +b_{\mathrm{h}}^{2}\left|\tilde{M}_{y}\right|^{2} \sin ^{2} \Theta \cos ^{2} \Theta \\
& -b_{\mathrm{h}}^{2}\left(\tilde{M}_{y} \tilde{M}_{z}^{*}+\tilde{M}_{z} \tilde{M}_{y}^{*}\right) \sin ^{3} \Theta \cos \Theta \\
& \mp b_{\mathrm{h}}\left(\tilde{N} \tilde{M}_{z}^{*}+\tilde{N}^{*} \tilde{M}_{z}\right) \sin ^{2} \Theta \\
& \pm b_{\mathrm{h}}\left(\tilde{N} \tilde{M}_{y}^{*}+\tilde{N}^{*} \tilde{M}_{y}\right) \sin \Theta \cos \Theta,
\end{aligned}
$$

Here $\Theta$ is the angle between the scattering vector $\vec{q}$ and the magnetic field $\vec{H}$ and $b_{\mathrm{h}}=$ $2.7 \cdot 10^{-15} \mathrm{~m} / \mu_{\mathrm{B}}$, where $\mu_{\mathrm{B}}$ is the Bohr magneton. Moreover, $\tilde{N}(\vec{q})$ and $\tilde{M}_{y, z}(\vec{q})$ denote the Fourier transforms of the nuclear scattering length density and of the magnetization in the $y$ - and $z$-directions, respectively. From the measured nsf intensities, the purely nuclear cross sections $I_{\text {nuc }}(q) \propto|\tilde{N}|^{2}$ and the nuclear magnetic cross terms $I_{\text {cross }}(q) \propto\left(\tilde{N} \tilde{M}_{z}^{*}+\tilde{N}^{*} \tilde{M}_{z}\right)$ 
were determined as a function of the applied field. For dilute isotropic particles and without formation of aggregates in magnetic field, no field dependence of the nuclear cross section is expected and hence the SANS signal should be qualitatively identical to the SAXS intensity. The same applies for the nuclear magnetic cross terms when the particles are homogeneously magnetized, such that the functional form of $\tilde{N}$ and $\tilde{M}_{z}$ would be identical.
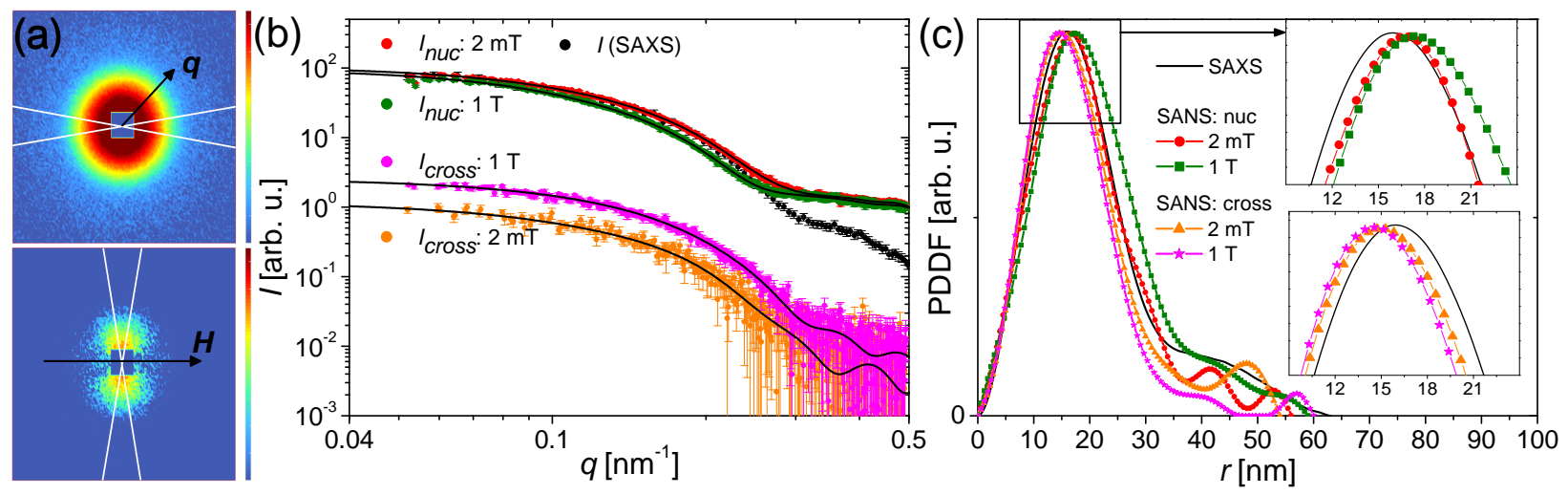

Figure 5: Polarized SANS experiments performed on the colloidal dispersion at $T=300 \mathrm{~K}$. (a) 2D scattering pattern of the nsf SANS intensity $I^{--}(\vec{q})$ (top) and of the difference $I^{--}(\vec{q})-I^{++}(\vec{q})$ (bottom), at $\mu_{0} H=2 \mathrm{mT}$. (b) SAXS scattering intensity $I(q)$ from Fig. 2 (rescaled), nuclear scattering intensity $I_{\text {nuc }}(q)$ determined by integrating the SANS intensity $I^{--}(q)$ in sectors parallel to $\vec{H}$ as well as the nuclear magnetic cross terms $I_{\text {cross }}(q)$ determined by sector average $I^{--}(\vec{q})-I^{++}(\vec{q})$ vertical to $\vec{H}$ for $\mu_{0} H=2 \mathrm{mT}$ and $\mu_{0} H=1 \mathrm{~T}$ (cross terms are rescaled by factor 0.05). The solid lines are the reconstructed curves for the corresponding PDDFs. (c) PDDFs determined by indirect Fourier transform of $I(q), I_{\text {nuc }}(q)$ and $I_{\text {cross }}(q)$. For comparison purposes the distributions are rescaled. Insets show the zoom of the peak maximums.

Fig. 5(a) shows the 2D scattering patterns of the nsf intensity $I^{--}(\vec{q})$ and of the difference $I^{--}(\vec{q})-I^{++}(\vec{q})$, measured at $\mu_{0} H=2 \mathrm{mT}$. Integration of $I^{--}(\vec{q})$ in a $10^{\circ}$ sector around $\Theta=0^{\circ}$ (Fig. 5(a), parallel to $\vec{H}$ ) enables determination of the purely nuclear 1D scattering intensity $I_{\text {nuc }}(q) \propto|\tilde{N}|^{2}$ of the nanoflowers, plotted in Fig. 5(b). Comparison with the SAXS intensity $I(q)$ from Fig. 2 (rescaled) shows that in the low $q$-range the shapes of both intensities are virtually identical. In the high $q$-range the two SANS scattering intensities differ due to the large incoherent scattering background from $\mathrm{H}_{2} \mathrm{O}$. An indirect Fourier transform ${ }^{40}$ of $I_{\text {nuc }}(q)$ at $\mu_{0} H=2 \mathrm{mT}$ results in a PDDF comparable to SAXS with the maximum of the main peak at $r=16.3 \mathrm{~nm}$ (Fig. $5(\mathrm{c})$ ). Interestingly, at $\mu_{0} H=1 \mathrm{~T}$ the determined intensity 
$I_{\text {nuc }}(q)$ is slightly shifted to lower values on the $q$ axis. As a result, the extracted PDDF is shifted to larger $r$ values with its maximum at $r=17.1 \mathrm{~nm}$. With TEM we observed that the particles are slightly anisotropic. Hence, we can conclude from SANS that the nanoflowers seem to be preferentially magnetized along their longest dimension due to the shape anisotropy, and that magnetic saturation is accompanied by a physical rotation of the nanoflowers in field direction. To gain information about the spatial magnetization configuration within the nanoflowers, the nuclear magnetic cross-term $I_{\text {cross }}(q) \propto\left(\tilde{N} \tilde{M}_{z}^{*}+\tilde{N}^{*} \tilde{M}_{z}\right)$ was determined at $\mu_{0} H=2 \mathrm{mT}$ and $1 \mathrm{~T}$. For this purpose the difference $I^{--}(\vec{q})-I^{++}(\vec{q})$ was integrated in a $10^{\circ}$ sector around $\Theta=90^{\circ}$ (Fig. 5(a), perpendicular to $\vec{H}$ ). For homogeneously magnetized, single-domain particles, the functional form of $\tilde{N}$ and $\tilde{M}_{z}$ are identical, at least if the surfactant layer is contrast matched by the matrix $\left(\mathrm{H}_{2} \mathrm{O}\right)$. Then $I_{\text {cross }}(q)$ would be expected to follow the purely nuclear scattering intensity of the iron oxide particles. As seen in Fig. $5(\mathrm{~b})$, the absolute values of $I_{\text {cross }}(q)$ measured at $1 \mathrm{~T}$ are a factor of about 2.4 times those at $2 \mathrm{mT}$. This is in excellent agreement with the isothermal magnetization curve (Fig. 4(a)) where the magnetization measured at $1 \mathrm{~T}\left(108 \mathrm{Am}^{2} / \mathrm{kg}_{\mathrm{Fe}}\right)$ is also about 2.4 times that at $2 \mathrm{mT}\left(46 \mathrm{Am}^{2} / \mathrm{kg}_{\mathrm{Fe}}\right)$. The functional forms of both cross terms are basically identical and comparable to that for nuclear scattering. Consequently, the PDDFs determined by an indirect Fourier transform ${ }^{40}$ of the cross sections $I_{\text {cross }}(q)$ are very similar to the purely nuclear PDDFs (Fig. $5(\mathrm{c})$ ). Only the maximum of the primary (main) peak is shifted in both cases by about 1-2 $\mathrm{nm}$ to lower values, which can be attributed to the slight shape anisotropy of the nanoflowers. Hence, we can directly conclude from the extracted PDDFs that the magnetic moments within the whole nanoflower volume were in fact oriented preferentially parallel to each other, as (indirectly) deduced before from the isothermal and optomagnetic measurements. 


\section{Relaxation dynamics of the nanoflowers}

Fig. 6(a) shows the normalized MRX signal $B(t) / c_{\max }$ of the colloidal dispersion and Fig. 6(b) shows the corresponding measurements of the real and imaginary parts of the AC susceptibility. Here, $c_{\max }$ is a normalization constant of the MRX signal, which is included to facilitate the simultaneous numerical inversion of the two data sets, and discussed further in the SI. The real part $\chi^{\prime}(\omega)$ of the susceptibility starts in the low-frequency range at a nearly constant value of $\chi_{0}=0.134$ (Fig. $6(\mathrm{~b})$ ). This concurs with the isothermal magnetization results in the previous section. A linear fit of $M(H)$ in units $[\mathrm{A} / \mathrm{m}]$ in the field range $H=0-1625 \mathrm{~A} / \mathrm{m}\left(\mu_{0} H=0-2 \mathrm{mT}\right)$ results in $\chi_{0}=0.151$. When $\omega>1000 \mathrm{~s}^{-1}, \chi^{\prime}(\omega)$ is

drastically reduced, but it does not reach zero within the experimentally accessible frequency range. Correspondingly, the imaginary part $\chi^{\prime \prime}(\omega)$ exhibits a pronounced peak with its maximum at $\omega \approx 31 \cdot 10^{3} \mathrm{~s}^{-1}$. Together, these observations indicate that the majority of the relaxation processes take place in the measured region $\omega=62.8 \mathrm{~s}^{-1}$ to $6.28 \cdot 10^{6} \mathrm{~s}^{-1}$. Due to the fact that MRX covers a time range of $t=0.28 \mathrm{~ms}$ to $1.5 \mathrm{~s}$, i.e., slow relaxation processes, the MRX signal $B(t) / c_{\max }$ (Fig. 6(a)) is already at the first data point much lower than $\chi_{0}$. From the combined susceptibility and MRX measurements, the underlying relaxation time distribution $P(\tau)=\chi_{0} p_{\mu}(\tau) \Delta \tau$ (where $p_{\mu}(\tau)$ is the moment-weighted probability density) was extracted by numerical inversion (Fig. 6(c)), with further details provided in the SI. In the window of experimental observation times, the relaxation time distribution $P(\tau)$ exhibits two distinct peaks, labeled 1 and 2 . 

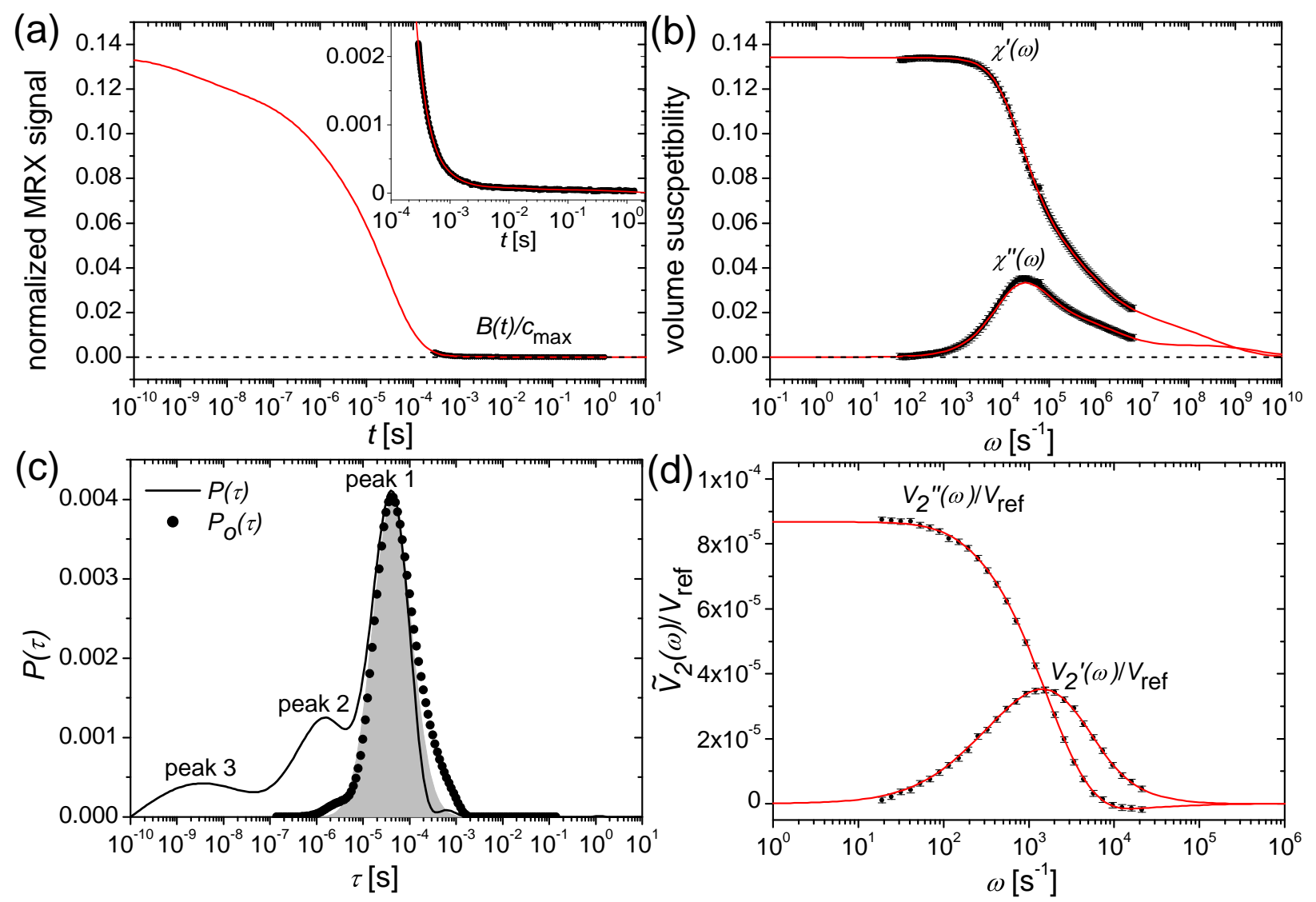

Figure 6: MRX, $\operatorname{ACS}(\omega)$ and frequency dependent optomagnetic measurements of the colloidal dispersion performed at $T=300 \mathrm{~K}$. (a) MRX: Normalized fluxgate signal of the colloidal dispersion $\left(B(t) / c_{\max }\right)$ and the reconstructed curve for $P(\tau)$ (solid line). (b) $\operatorname{ACS}(\omega)$ : Real and imaginary part of the measured AC susceptibility of the colloidal dispersion $\left(\chi^{\prime}(\omega)\right.$, $\left.\chi^{\prime \prime}(\omega)\right)$ and the reconstructed curves for $P(\tau)$ (solid lines). (c) Relaxation time distribution $P(\tau)=\chi_{0} p_{\mu}(\tau) \Delta \tau$ (where $p_{\mu}(\tau)$ is the moment weighted probability density) determined by the numerical inversion of the $\operatorname{ACS}(\omega)$ and MRX data, and the rescaled distribution $P_{\mathrm{o}}(\tau)$ extracted from the optomagnetic measurements. Grey area: Rescaled distribution $p_{V}\left(\tau_{\mathrm{B}}\right) \Delta \tau_{\mathrm{B}}$ (logarithmic scaling $\Delta \tau_{\mathrm{B}}$ ), calculated for a particle ensemble with a volume-weighted lognormal distribution of the hydrodynamic size $\left(\sigma=0.32,\left\langle D_{\mathrm{h}}\right\rangle=54 \mathrm{~nm}\right)$. (d) Normalized signal $\tilde{V}_{2}(\omega) / V_{\text {ref }}$ of the optomagnetic measurements and reconstructed curves (solid lines) for the extracted relaxation time distribution $P_{\mathrm{o}}(\tau)$. 
Optomagnetic measurements vs. frequency at low magnetic field amplitude were used to assess the hydrodynamic size of the particles as described in detail by Fock et al. ${ }^{33}$. In Fig. $6(\mathrm{~d})$ the real and imaginary part of the normalized second harmonic signal $\tilde{V}_{2}(\omega) / V_{\text {ref }}$ are plotted. Optomagnetic measurements are sensitive only to Brownian relaxation of the slightly elongated nanoflowers and are completely insensitive to relaxation arising from Néel processes. Theoretically, at low magnetic field amplitudes, the signal $\tilde{V}_{2}(\omega) / V_{\text {ref }} \propto i \tilde{\chi}(\omega)^{2}$, with $\tilde{\chi}(\omega)$ being the complex susceptibility. ${ }^{33}$ Therefore, the distribution of Brownian relaxation times can be extracted by a combined numerical inversion of $V_{2}^{\prime}(\omega) / V_{\text {ref }}$ and $V_{2}^{\prime \prime}(\omega) / V_{\text {ref }}$, in analogy to the inversion of only the ACS data, and is plotted in Fig. 6(c).

A comparison of $P_{\mathrm{o}}(\tau)$ with the distribution $P(\tau)$, extracted from both the ACS $(\omega)$ and MRX data, indicate that there is excellent agreement between the two techniques and thus we attribute this peak to Brownian rotation. To derive the distribution of hydrodynamic sizes, a lognormal distribution of relaxation times was fitted to peak 1 (grey area in Fig. 6(c)) and related to the distribution of hydrodynamic sizes using Eq. (3). The distribution has a mean value of $\left\langle D_{\mathrm{h}}\right\rangle=54 \mathrm{~nm}$ and $\sigma=0.32$. This is in good agreement with the hydrodynamic size measured by DLS $\left(\left\langle D_{\mathrm{h}}\right\rangle=56 \mathrm{~nm}\right)$.

Peaks 2 and 3 (Fig. 6(c), lower $\tau$ ), on the other hand, are not observed by optomagnetic measurements and therefore must arise from a Néel-like relaxation. As shown in the SI, similar spectra were also determined by numerical inversion of two other $\operatorname{ACS}(\omega)$ data sets, which emphasizes that at least peak 2 is reproducible. The maximum of peak 2 is located at about $\tau=10^{-6} \mathrm{~s}$, which is in good agreement with the estimated Néel relaxation times (Eq. 2) calculated for $2 K V / k_{\mathrm{B}}=4700 \pm 300 \mathrm{~K}$ (as determined previously by Mössbauer spectroscopy). Peak 3, observed outside the window of experimental observation times, cannot be given a direct physical interpretation as it may be a numerical artifact resulting from the boundary condition on the distribution of relaxation times at low $\tau$. Nevertheless, the finite value of the susceptibility at $\omega=6.28 \cdot 10^{6} \mathrm{~s}^{-1}\left(\chi^{\prime \prime}=0.009\right)$ clearly shows the presence of fast relaxation processes $\left(\tau<1.6 \cdot 10^{-7} \mathrm{~s}\right)$ and we assume that these may be 
connected to the disordered/uncorrelated spins within the nanoflowers, which were observed by isothermal magnetometry (Fig. 4(b), low moment contributions).

\section{Magnetic hyperthermia performance of the nanoflowers}

In Table 1 we list the intrinsic loss power $\left(\operatorname{ILP}^{56}\right)$ values measured of the colloidal dispersion for varying viscosity. As can be seen, the ILP values are nearly independent of the viscosity and with values of about $7 \mathrm{nHm}^{2} / \mathrm{kg}_{\mathrm{Fe}}$, they are quite large compared to literature, where typical values are in the range $0.2-4.4 \mathrm{nHm}^{2} / \mathrm{kg}_{\mathrm{Fe}} \cdot{ }^{56}$ The insensitivity to the viscosity confirms that the heating in the nanoflowers is generated by internal magnetization processes and not by Brownian relaxation. This is consistent with our analysis of the relaxation dynamics which shows that Brownian relaxation takes place at frequencies much smaller than $\omega=5.9 \cdot 10^{6} \mathrm{~s}^{-1}$ and that the relaxation dynamics of the nanoflowers is dominated by internal moment relaxation in the high-frequency range.

To complete, we compare the average measured ILP value (about $7 \mathrm{nHm}^{2} / \mathrm{kg}_{\mathrm{Fe}}$ ) with the expected value according to the LRT. In this theory, the ILP value is given by:

$$
\mathrm{ILP}=\frac{2 \pi P_{d}}{c_{\mathrm{Fe}} H^{2} \omega}=\frac{\pi \mu_{0} \chi^{\prime \prime}(\omega)}{c_{\mathrm{Fe}}}
$$

where $P_{d}$ is the dissipated power density (Eq. (1)) and $c_{\mathrm{Fe}}$ is the iron concentration in the colloid; in this case determined by ICP-OES to $c_{\mathrm{Fe}}=5 \mathrm{~kg}_{\mathrm{Fe}} / \mathrm{m}^{3}$. At $\omega=6.28 \cdot 10^{6} \mathrm{~s}^{-1}$ the measured imaginary part of the susceptibility was $\chi^{\prime \prime}=0.009$ and hence the ILP value expected to be about $7.1 \mathrm{nHm}^{2} / \mathrm{kg}_{\mathrm{Fe}}$. The good agreement between measured and calculated ILP value indicates that the LRT properly describes the heating behavior of the nanoflowers.

Table 1: ILP values measured for the colloidal dispersion of nanoflowers $\left(\mu_{0} H=\right.$ $\left.8.8 \mathrm{mT}, \omega=5.9 \cdot 10^{6} \mathrm{~s}^{-1}\right)$ with varying glycerol concentration and hence viscosity.

\begin{tabular}{lccccc}
\hline glycerol content [wt\%] & 0 & 18.75 & 37.5 & 56.25 & 75 \\
viscosity $[\mathrm{mPa} \cdot \mathrm{s}]$ & 1 & 2.2 & 4.1 & 7.5 & 16.1 \\
$\mathrm{ILP}\left[\mathrm{nHm} / \mathrm{kg}_{\mathrm{Fe}}\right]$ & $7.4 \pm 0.1$ & $7.1 \pm 0.1$ & $6.2 \pm 0.1$ & $6.2 \pm 0.1$ & $6.9 \pm 0.1$ \\
\hline
\end{tabular}


With this in mind, we conclude that the dominant heating mechanism are significant intrinsic moment relaxations, probably enhanced by the internal spin disorder.

\section{Conclusions}

We presented here an in-depth study relating the structure of nanoflowers to their magnetic properties (in timescales spanning from quasi-static to microseconds) and finally to the remarkable heat generated by magnetic hyperthermia. The particles had a mean size of about $39 \mathrm{~nm}$, were composed of several iron oxide crystallites/cores, and as a result of the nanocrystalline structure had an irregular shape and a slight shape anisotropy. We demonstrated that the individual nanoflowers had a remanent magnetization (due to an exchange coupling between the cores), were preferentially magnetized along their longest dimension (due to shape anisotropy), but with a slight internal spin disorder/misalignment.

We determined the relaxation time distribution of the colloidally dispersed nanoflowers, which exhibited two distinct peaks within the experimentally accessible time range. We could associate the peak of large relaxation times (slow relaxation processes) with Brownian relaxation, and the derived hydrodynamic size distribution was in good agreement with DLS measurements. The peak in the small relaxation time range could be then attributed to classical Néel-like relaxation processes. However, even at the largest measured frequency the volume susceptibility still had finite values indicating the presence of very fast relaxation processes outside of the accessible time range, which we attribute to the disordered/uncorrelated spins within the individual nanoflowers.

Finally, we showed that the intrinsic loss power of the nanoflowers with a value of about $7 \mathrm{nHm}^{2} / \mathrm{kg}_{\mathrm{Fe}}$ was high compared to conventional iron oxide nanoparticles and also independent of the viscosity of the surrounding medium. The insensitivity to viscosity agreed well with the observation that in the high-frequency range the relaxation dynamics of the nanoflowers was dominated by internal moment relaxation. The average detected ILP value 
could be well described within the linear response theory, and hence the large detected ILP values could be directly connected to the quite large value of the imaginary part of the volume susceptibility at high frequencies. We surmise that the observed fast relaxation dynamics, which resulted in finite values of $\chi^{\prime \prime}$ also at high frequencies, is related to the fact that the nanoflowers constitute disordered spin systems caused by the nanocrystalline structure, but which are easily magnetized by external magnetic fields likely aided by an exchange coupling. By using kinetic Monte-carlo simulations ${ }^{57,58}$ of the nanoflower particle structures, we verified that including exchange interactions between the cores in the multicore structure together with anisotropy energy and dipole-dipole interactions, increases the power absorption significantly.

\section{Acknowledgments}

The authors thank the Institute Laue-Langevin for provision of neutron beamtime at the instrument D33. This project has received funding from the European Commission Framework Programme 7 under grant agreement no 604448 (NanoMag).

\section{Supporting Information Available}

The following files are available free of charge.

- Video displaying 3D TEM tomography of the nanoflowers.

- Text file with details regarding the combined numerical inversion of $\operatorname{ACS}(\omega)$ and MRX data, additional $\operatorname{ACS}(\omega)$ measurements and results from Mössbauer spectroscopy. 


\section{References}

(1) Pankhurst, Q. A.; Connolly, J.; Jones, S. K.; Dobson, J. Applications of magnetic nanoparticles in biomedicine. J. Phys. D: Appl. Phys. 2003, 36, R167.

(2) Gupta, A. K.; Gupta, M. Synthesis and surface engineering of iron oxide nanoparticles for biomedical applications. Biomaterials 2005, 26, 3995-4021.

(3) Laurent, S.; Forge, D.; Port, M.; Roch, A.; Robic, C.; Vander Elst, L.; Muller, R. N. Magnetic iron oxide nanoparticles: synthesis, stabilization, vectorization, physicochemical characterizations, and biological applications. Chem. Rev. 2008, 108, 2064-2110.

(4) Wu, W.; Wu, Z.; Yu, T.; Jiang, C.; Kim, W.-S. Recent progress on magnetic iron oxide nanoparticles: synthesis, surface functional strategies and biomedical applications. Sci. Technol. Adv. Mater. 2015, 16, 023501.

(5) Jordan, A.; Scholz, R.; Wust, P.; Fähling, H.; Felix, R. Magnetic fluid hyperthermia (MFH): Cancer treatment with AC magnetic field induced excitation of biocompatible superparamagnetic nanoparticles. J. Magn. Magn. Mater. 1999, 201, 413-419.

(6) Kozissnik, B.; Bohorquez, A. C.; Dobson, J.; Rinaldi, C. Magnetic fluid hyperthermia: Advances, challenges, and opportunity. Int. J. Hyperthermia 2013, 29, 706-714.

(7) Deatsch, A. E.; Evans, B. A. Heating efficiency in magnetic nanoparticle hyperthermia. J. Magn. Magn. Mater. 2014, 354, 163-172.

(8) Blanco-Andujar, C.; Ortega, D.; Southern, P.; Pankhurst, Q.; Thanh, N. High performance multi-core iron oxide nanoparticles for magnetic hyperthermia: microwave synthesis, and the role of core-to-core interactions. Nanoscale 2015, 7, 1768-1775.

(9) Andreu, I.; Natividad, E.; Solozábal, L.; Roubeau, O. Nano-objects for addressing the control of nanoparticle arrangement and performance in magnetic hyperthermia. $A C S$ Nano 2015, 9, 1408-1419. 
(10) Conde-Leboran, I.; Baldomir, D.; Martinez-Boubeta, C.; Chubykalo-Fesenko, O.; del Puerto Morales, M.; Salas, G.; Cabrera, D.; Camarero, J.; Teran, F. J.; Serantes, D. A single picture explains diversity of hyperthermia response of magnetic nanoparticles. $J$. Phys. Chem. C 2015, 119, 15698-15706.

(11) Nemati, Z.; Alonso, J.; Martinez, L.; Khurshid, H.; Garaio, E.; Garcia, J.; Phan, M.; Srikanth, H. Enhanced magnetic hyperthermia in iron oxide nano-octopods: size and anisotropy effects. J. Phys. Chem. C 2016, 120, 8370-8379.

(12) Muela, A.; Muñoz, D.; Martín-Rodríguez, R.; Orue, I.; Garaio, E.; Abad Díaz de Cerio, A.; Alonso, J.; García, J. A.; Fdez-Gubieda, M. L. Optimal parameters for hyperthermia treatment using biomineralized magnetite nanoparticles: theoretical and experimental approach. J. Phys. Chem. C 2016, 120, 24437-24448.

(13) Ovejero, J. G.; Cabrera, D.; Carrey, J.; Valdivielso, T.; Salas, G.; Teran, F. J. Effects of inter-and intra-aggregate magnetic dipolar interactions on the magnetic heating efficiency of iron oxide nanoparticles. Phys. Chem. Chem. Phys. 2016, 18, 10954-10963.

(14) van Raap, M. F.; Coral, D.; Yu, S.; Muñoz, G.; Sánchez, F.; Roig, A. Anticipating hyperthermic efficiency of magnetic colloids using a semi-empirical model: a tool to help medical decisions. Phys. Chem. Chem. Phys. 2017, 19, 7176-7187.

(15) Rosensweig, R. E. Heating magnetic fluid with alternating magnetic field. J. Magn. Magn. Mater. 2002, 252, 370-374.

(16) Debye, P. Polar Molecules; New York: Dover, 1945.

(17) Leliaert, J.; Coene, A.; Crevecoeur, G.; Vansteenkiste, A.; Eberbeck, D.; Wiekhorst, F.; Van Waeyenberge, B.; Dupré, L. Regarding the Néel relaxation time constant in magnetorelaxometry. J. Appl. Phys. 2014, 116, 163914. 
(18) Fock, J.; Hansen, M. F.; Frandsen, C.; Mørup, S. On the interpretation of Mössbauer spectra of magnetic nanoparticles. J. Magn. Magn. Mater. 2018, 445, 11-21.

(19) Doaga, A.; Cojocariu, A.; Amin, W.; Heib, F.; Bender, P.; Hempelmann, R.; Caltun, O. Synthesis and characterizations of manganese ferrites for hyperthermia applications. Mater. Chem. Phys. 2013, 143, 305-310.

(20) Mazario, E.; Menéndez, N.; Herrasti, P.; Cañete, M.; Connord, V.; Carrey, J. Magnetic hyperthermia properties of electrosynthesized cobalt ferrite nanoparticles. J. Phys. Chem. C 2013, 117, 11405-11411.

(21) Casula, M. F.; Conca, E.; Bakaimi, I.; Sathya, A.; Materia, M. E.; Casu, A.; Falqui, A.; Sogne, E.; Pellegrino, T.; Kanaras, A. G. Manganese doped-iron oxide nanoparticle clusters and their potential as agents for magnetic resonance imaging and hyperthermia. Phys. Chem. Chem. Phys. 2016, 18, 16848-16855.

(22) Maity, D.; Chandrasekharan, P.; Pradhan, P.; Chuang, K.-H.; Xue, J.-M.; Feng, S.-S.; Ding, J. Novel synthesis of superparamagnetic magnetite nanoclusters for biomedical applications. J. Mater. Chem. 2011, 21, 14717-14724.

(23) Hugounenq, P.; Levy, M.; Alloyeau, D.; Lartigue, L.; Dubois, E.; Cabuil, V.; Ricolleau, C.; Roux, S.; Wilhelm, C.; Gazeau, F. et al. Iron oxide monocrystalline nanoflowers for highly efficient magnetic hyperthermia. J. Phys. Chem. C 2012, 116, 15702-15712.

(24) Lartigue, L.; Hugounenq, P.; Alloyeau, D.; Clarke, S. P.; Lévy, M.; Bacri, J.-C.; Bazzi, R.; Brougham, D. F.; Wilhelm, C.; Gazeau, F. Cooperative organization in iron oxide multi-core nanoparticles potentiates their efficiency as heating mediators and MRI contrast agents. ACS Nano 2012, 6, 10935-10949.

(25) Dutz, S. Are Magnetic Multicore Nanoparticles Promising Candidates for Biomedical Applications? IEEE Trans. Magn. 2016, 52, 1-3. 
(26) Gavilán, H.; Kowalski, A.; Heinke, D.; Sugunan, A.; Sommertune, J.; Varón, M.; Bogart, L. K.; Posth, O.; Zeng, L.; González-Alonso, D. et al. Colloidal Flower-Shaped Iron Oxide Nanoparticles: Synthesis Strategies and Coatings. Part. Part. Syst. Charact. 2017, 34, 1700094.

(27) Fock, J.; Bogart, L. K.; Posth, O.; Hansen, M. F.; Pankhurst, Q. A.; Frandsen, C. Uncertainty budget for determinations of mean isomer shift from Mössbauer spectra. Hyperfine Interact. 2016, 237, 23.

(28) Dewhurst, C. D.; Grillo, I.; Honecker, D.; Bonnaud, M.; Jacques, M.; Amrouni, C.; Perillo-Marcone, A.; Manzin, G.; Cubitt, R. The small-angle neutron scattering instrument D33 at the Institut Laue-Langevin. J. Appl. Crystallogr. 2016, 49, 1-14.

(29) Bender, P.; Fernández Barquín, L.; Fdez-Gubieda, M.-L.; González-Alonso, D.; Honecker, D.; Marcano, L.; Szczerba, W. Spin correlation in clusters of magnetic nanoparticles. Institut Laue-Langevin (ILL) 2017, Doi: 10.5291/ILL-DATA.5-53-267.

(30) Honecker, D.; Ferdinand, A.; Döbrich, F.; Dewhurst, C.; Wiedenmann, A.; GómezPolo, C.; Suzuki, K.; Michels, A. Longitudinal polarization analysis in small-angle neutron scattering. Eur. Phys. J. B 2010, 76, 209-213.

(31) Ludwig, F.; Heim, E.; Schilling, M.; Enpuku, K. Characterization of superparamagnetic $\mathrm{Fe}_{3} \mathrm{O}_{4}$ nanoparticles by fluxgate magnetorelaxometry for use in biomedical applications. J. Appl. Phys. 2008, 103, 07A314.

(32) Ludwig, F.; Guillaume, A.; Schilling, M.; Frickel, N.; Schmidt, A. M. Determination of core and hydrodynamic size distributions of $\mathrm{CoFe}_{2} \mathrm{O}_{4}$ nanoparticle suspensions using ac susceptibility measurements. J. Appl. Phys. 2010, 108, 033918.

(33) Fock, J.; Jonasson, C.; Johansson, C.; Hansen, M. F. Characterization of fine particles using optomagnetic measurements. Phys. Chem. Chem. Phys. 2017, 19, 8802-8814. 
(34) Wildeboer, R.; Southern, P.; Pankhurst, Q. On the reliable measurement of specific absorption rates and intrinsic loss parameters in magnetic hyperthermia materials. $J$. Phys. D: Appl. Phys. 2014, 47, 495003.

(35) Berkov, D.; Görnert, P.; Buske, N.; Gansau, C.; Mueller, J.; Giersig, M.; Neumann, W.; $\mathrm{Su}, \mathrm{D}$. New method for the determination of the particle magnetic moment distribution in a ferrofluid. J. Phys. D: Appl. Phys. 2000, 33, 331.

(36) Enpuku, K.; Tanaka, T.; Tamai, Y.; Dang, F.; Enomoto, N.; Hojo, J.; Kanzaki, H.; Usuki, N. Size distribution of magnetic marker estimated from AC susceptibility in solution for biosensor application. Jpn. J. Appl. Phys. 2008, 47, 7859.

(37) Hansen, S. Estimation of the relaxation spectrum from dynamic experiments using Bayesian analysis and a new regularization constraint. Rheol. Acta 2008, 47, 169-178.

(38) van Rijssel, J.; Kuipers, B. W.; Erné, B. H. Non-regularized inversion method from light scattering applied to ferrofluid magnetization curves for magnetic size distribution analysis. J. Magn. Magn. Mater. 2014, 353, $110-115$.

(39) Leliaert, J.; Schmidt, D.; Posth, O.; Liebl, M.; Eberbeck, D.; Coene, A.; Steinhoff, U.; Wiekhorst, F.; Waeyenberge, B. V.; Dupré, L. Interpreting the magnetorelaxometry signal of suspended magnetic nanoparticles with Kaczmarz' algorithm. J. Phys. D: Appl. Phys. 2017, 50, 195002.

(40) Bender, P.; Bogart, L. K.; Posth, O.; Szczerba, W.; Rogers, S. E.; Castro, A.; Nilsson, L.; Zeng, L. J.; Sugunan, A.; Sommertune, J. et al. Structural and magnetic properties of multi-core nanoparticles analysed using a generalised numerical inversion method. Sci. Rep. 2017, 7, 45990.

(41) Bender, P.; Balceris, C.; Ludwig, F.; Posth, O.; Bogart, L.; Szczerba, W.; Castro, A.; Nilsson, L.; Costo, R.; Gavilán, H. et al. Distribution functions of magnetic nanoparticles determined by a numerical inversion method. New J. Phys 2017, 19, 073012. 
(42) Hansen, S. Bayesian estimation of hyperparameters for indirect Fourier transformation in small-angle scattering. J. Appl. Crystallogr. 2000, 33, 1415-1421.

(43) Vestergaard, B.; Hansen, S. Application of Bayesian analysis to indirect Fourier transformation in small-angle scattering. J. Appl. Crystallogr. 2006, 39, 797-804.

(44) Glatter, O. A new method for the evaluation of small-angle scattering data. J. Appl. Crystallogr. 1977, 10, 415-421.

(45) Glatter, O. The interpretation of real-space information from small-angle scattering experiments. J. Appl. Crystallogr. 1979, 12, 166-175.

(46) Fock, J.; Bogart, L. K.; González-Alonso, D.; Espeso, J. I.; Hansen, M. F.; Varón, M.; Frandsen, C.; Pankhurst, Q. A. On the centre of gravity method for measuring the composition of magnetite/maghemite mixtures, or the stoichiometry of magnetitemaghemite solid solutions, via 57Fe Mössbauer spectroscopy. J. Phys. D: Appl. Phys 2017, 50, 265005.

(47) Mørup, S. Magnetic hyperfine splitting in mössbauer spectra of microcrystals. J. Magn. Magn. Mater. 1983, 37, 39-50.

(48) Dormann, J.; D’Orazio, F.; Lucari, F.; Tronc, E.; Prené, P.; Jolivet, J.; Fiorani, D.; Cherkaoui, R.; Nogues, M. Thermal variation of the relaxation time of the magnetic moment of $\gamma-\mathrm{Fe}_{2} \mathrm{O}_{3}$ nanoparticles with interparticle interactions of various strengths. Phys. Rev. B 1996, 53, 14291.

(49) Jonsson, T.; Nordblad, P.; Svedlindh, P. Dynamic study of dipole-dipole interaction effects in a magnetic nanoparticle system. Phys. Rev. B 1998, 57, 497.

(50) Pisane, K.; Despeaux, E.; Seehra, M. Magnetic relaxation and correlating effective magnetic moment with particle size distribution in maghemite nanoparticles. J. Magn. Magn. Mater. 2015, 384, 148-154. 
(51) Chuev, M. A.; Cherepanov, V. M.; Polikarpov, M. A. On the shape of the gamma resonance spectra of slowly relaxing nanoparticles in a magnetic field. JETP Lett. 2010, 92, 21-27.

(52) Komorida, Y.; Mito, M.; Deguchi, H.; Takagi, S.; Millán, A.; Silva, N. J. O.; Palacio, F. Surface and core magnetic anisotropy in maghemite nanoparticles determined by pressure experiments. Appl. Phys. Lett. 2009, 94 .

(53) Figueroa, A.; Bartolomé, J.; García, L.; Bartolomé, F.; Arauzo, A.; Millán, A.; Palacio, F. Magnetic Anisotropy of Maghemite Nanoparticles Probed by RF Transverse Susceptibility. Phys. Procedia 2015, 75, 1050 - 1057, 20th International Conference on Magnetism, ICM 2015.

(54) Coey, J. M. D. Magnetism and Magnetic Materials; Cambridge University Press, 2010.

(55) Michels, A. Magnetic small-angle neutron scattering of bulk ferromagnets. J. Phys.: Condens. Matter 2014, 26, 383201.

(56) Kallumadil, M.; Tada, M.; Nakagawa, T.; Abe, M.; Southern, P.; Pankhurst, Q. A. Suitability of commercial colloids for magnetic hyperthermia. J. Magn. Magn. Mater. 2009, 321, 1509-1513.

(57) Andersson, J.-O.; Djurberg, C.; Jonsson, T.; Svedlindh, P.; Nordblad, P. Monte Carlo studies of the dynamics of an interacting monodispersive magnetic-particle system. Phys. Rev. B 1997, 56, 13983.

(58) Du, H.; Du, A. Effect of exchange and dipolar interactions on the hysteresis of magnetic nanoparticle systems. phys. stat. sol. (b) 2007, 244, 1401-1408. 


\section{Graphical TOC Entry}

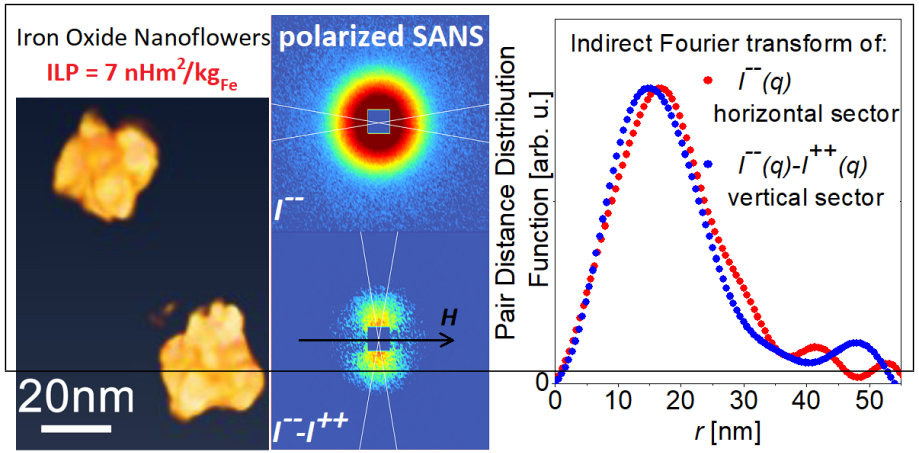

